mittee and eminent people in marine science and technology. The centre was authorized by the Minister for Science and Technology as the exclusive institute defined in the Marine Science and Technology Centre Act. The centre is accordingly to be financed both by industry and by the government.

The Japan Marine Science and Technology Centre (JAMSTEC) will primarily be concerned with the development of the advanced technology that will be required jointly in various aspects of marine development, the installation of large-scale research facilities for common use by the government, industry and the universities, and the education and training of scientists and technologists in advanced marine science and technology. The centre will be well equipped and will have a staff of more than 200 by 1975 .
Those Japanese industries with a marine bias take a keen interest in offshore oil and gas exploitation, civil engineering in coastal areas and, in the not too distant future, the exploitation of manganese nodules on the deep ocean floor.

Japanese shipyards are well equipped to build offshore drilling rigs of any size and for any purpose and shape. A semi-submersible rig called Hakuryu 2 was built last year; it can work at a depth of 200 metres and is capable of drilling down to 9,000 metres below the seafloor. Development work on a remotely controlled drilling rig capable of working on the seafloor at a depth of 250 to 300 metres and of drilling through 9,000 metres is being jointly carried out by several industries under contract with the Ministry of International Trade and Industry.

\title{
Amino-acid Production by the Fermentation Process
}

\section{SHUKUO KINOSHITA}

Kyowa Hakko Kogyo Limited, Tokyo

\section{The discovery in Japan of how to manufacture $l$ amino-acids with micro- organisms was a tremendous stimulus to research cooperation.}

EsTABLISHING a commercially applicable method for the synthesis of sodium glutamate has long been a subject of interest to chemists. Of the various problems involved, one of the most difficult was how to separate from the synthetic product, $d l$ glutamate, the one isomer, $l$ glutamate, that has the effect of enhancing the flavour of foods when added in appropriate amounts. I considered the whole problem myself while I was engaged in microbiological research, and came to the conclusion that microorganisms could well be useful.

The proteins of living cells contain $l$ amino-acids, which are biologically synthesized from carbohydrates and nitrogenous substances contained in the medium. But although living cells can manufacture $l$ amino-acids, these are vital to the synthesis of their own cellular proteins, and so would not be expected to be available "on tap" for other purposes.

There was nothing in literature at that time to suggest how amino-acids could be extracted from cells. And, anyway, how could the synthesis of glutamate be made to dominate the synthesis of other amino-acids?

Cellular synthesis of amino-acids is normally stably balanced, otherwise the process of protein synthesis would not work properly.

Some kind of change in favour of glutamate synthesis must clearly be made to occur if economic production of glutamate is to be feasible. Bearing in mind the difficulties, I started doing screening research on microorganisms from various sources, and in 1957 I discovered a novel species, later named Corynebacterium glutamicum, which seemed to suit my purposes perfectly.

When the bacteria were cultivated in an agitated medium containing glucose, ammonia and minerals at $p \mathrm{H} 7$, about $30 \mathrm{~g} \mathrm{l}^{-1}$ of glutamate was excreted into the medium by the cells. Other amino-acids, such as alanine, aspartate and glutamine, were often found in the mixture, but in negligible amounts. All glutamate produced by this microbiological method was definitely confirmed to be the $l$ form.

These results were surprising, and the method proved to be a most important breakthrough. It was clear at that time that there is some subtle mechanism operating which causes glutamate synthesis to become predominant in the cells and excretion to take place.

The key substance involved in the mechanism turned out to be biotin, and the amount of it present in the medium was found to be critical. Glutamate production occurs when the biotin content of the medium is 1 to 5 parts in $10^{9}$. At higher concentrations glutamate production is greatly reduced. These results were first reported at the International Symposium on Enzyme Chemistry in Tokyo and Kyoto (1957), and there were immediate repercussions in the form of a movement to establish a nation-wide research organization.

Microbiological research groups in universities and government research bodies were as a consequence organized in such a way that the basic and academic problems of amino-acid biosynthesis could be coherently studied. Special research funds were given to these groups by the government, and their common aim was called "basic research on amino-acid fermentation". Government support for these academic groups continued for three years, from 1957 to 1959 .

As a result of this financial support, the literature on fermentative production of amino-acids promptly mushroomed. The symposium on amino-acid fermentation was a natural outcome; it was first held in April 1959 and since then has taken place every spring and autumn. A body called the Association of Amino-acid Fermentation was established at the same time, both to organize the symposium and to publish the papers every six months. Financial support for the association comes from individual membership fees and from industrial companies as supporting members.

As a direct result of these activities, subjects ranging from basic ones like cell wall permeability to more practical ones like production control and process designs are now discussed at the symposium. Much biochemical work has been done during the past ten years or so, and a completely new technological field, fermentations by metabolic control, has been firmly established. Most of the $l$ amino-acids are now commercially available, and most are made by fermentation methods.

Another recent outstanding achievement has been nucleotide production by microbial means. Starting from the production of flavour-enhancing nucleotides such as inosinic acid and guanylic acid, various others like AMP, ADP, ATP, GDP, GTP, FAD and NAD can now be produced in commercial quantities.

The literature in the nucleotide field became so abundant, in fact, that the Association of Amino-acid Fermentation had to change its name to the Association of Amino-acid and Nucleic Acid Fermentation. 